\title{
On vector differential forms attached to automorphic forms.
}

\author{
Dedicated to Professor Z. Suetuna. \\ By Michio KugA and Goro ShImURA
}

(Received Sept. 18, 1959)

In recent works [2], [3], it was found that the integral of certain vector differential forms, attached to automorphic forms with respect to a Fuchsian group $G$, is important in the arithmetic theory of modular correspondences. Those vector differential forms $\omega$ are defined on the upper half plane and satisfy the transformation formula

$$
\omega \circ \sigma=M(\sigma) \omega
$$

for every element $\sigma$ of the group $G$, where $M(\sigma)$ is a tensor representation of $G$. The object of the present paper is to determine all holomorphic forms satisfying this relation (1). $M$ being of degree $2 m-1$, we can attach to every cusp form of degree $\leqq 2 m$ a holomorphic form $\omega$ with the representation $M$ (Theorem 1). Conversely, any holomorphic form satisfying (1) is expressed as a sum of the forms thus obtained from cusp forms of degree $\leqq 2 m$; and this expression gives a direct decomposition of the vector space $\mathfrak{F}$ of such holomorphic forms (Theorem 2). Hence the dimension of the vector space $\widetilde{F}$ is easily obtained if we know the dimension of the linear space of cusp forms for each degree. We note that the integral of the form attached to a cusp form of degree $<2 m$ has a period cohomologous to 0 , in the sense described in [3]. This fact distinguishes among such forms the forms attached to cusp forms of degree $2 m$, which were the object of the investigation in [3].

\section{$\S 1$. Cusp forms with respect to a Fuchsian group.}

Let $\mathscr{H}$ denote the upper half plane, the set of all complex numbers with positive imaginary parts. Every element $\sigma=\left(\begin{array}{ll}a & b \\ c & d\end{array}\right)$ of $S L(2, \boldsymbol{R})$ operates on $\mathcal{H}$, as usual :

$$
\sigma(z)=\frac{a z+b}{c z+d}
$$

we put

$$
J(\sigma, z)=(c z+d)^{-1}
$$


For every differential form $\omega$ on $\mathscr{H}$ we shall denote by $\omega \circ \sigma$ the transform of $\omega$ by $\sigma$; so if $\omega$ is expressed in the form $\omega=f(z) d z$ for a function $f(z)$ on $\mathscr{H}$, we have $\omega \circ \sigma=f(\sigma(z)) J(\sigma, z)^{2} d z$.

Let $G$ be a discrete subgroup of $S L(2, \boldsymbol{R})$ such that $S L(2, \boldsymbol{R}) / G$ has a finite total volume, measured by an invariant volume element. Then, $G$, as group of transformations on $\mathscr{G}$, is a Fuchsian group; namely, $G$ operates discontinuously on $\mathscr{H}$ and $\mathscr{H} / G$ has a fundamental domain $\mathscr{D}$ with a finite Poincaré area. If we denote by $\mathscr{H}^{*}$ the join of $\mathscr{H}$ and the "cusps" of $G$, the quotient space $\mathscr{H}^{*} / G$, with a suitable analytic structure, can be regarded as a compact Riemann surface.

A cusp of $G$ is the fixed point of a parabolic element of $G$, which is a real number or the point at infinity $\infty$. Let $s$ be a cusp of $G$. Put

$$
\rho=\left(\begin{array}{ll}
-s & 1 \\
-1 & 0
\end{array}\right) \text { or } \rho=\left(\begin{array}{ll}
1 & 0 \\
0 & 1
\end{array}\right)
$$

according as $s$ is a real number or $\infty$. Then the set of all elements of $G$ having $s$ as fixed point is the free cyclic group generated by an element $\tau$ of $G$, which is of the form

$$
\tau=\rho\left(\begin{array}{cc}
1 & h \\
0 & 1
\end{array}\right) \rho^{-1}
$$

where $h$ is a positive real number.

Let $\nu$ be an integer. We shall understand, by an automorphic form of degree $\nu$ with respect to $G$, a function $f(z)$ on $\mathscr{H}$ satisfying the following conditions (A 1-3).

(A 1) $f(z)$ is meromorphic on $\mathcal{H}$.

(A 2) For every $\sigma \in G$, we have $f(\sigma(z)) J(\sigma, z)^{\nu}=f(z)$.

Consider a cusp $s$ of $G$; the transformation $\rho$ and the positive number $h$ being defined for $s$ as above, we see that, if $f$ satisfies (A 1-2), the function $f(\rho(z)) J(\rho, z)^{\nu}$ is invariant under the translation $z \rightarrow z+h$. Hence, if we put

$$
q=\exp \left(2 \pi i h^{-1} z\right),
$$

there exists a function $g(q)$ meromorphic in the domain $0<|q|<1$ such that

$$
f(\rho(z)) J(\rho, z)^{\nu}=g(q) .
$$

The condition (A 3 ) is now stated as follows.

(A 3) For every cusp s of $G$, the function $g(q)$, defined as above, is meromorphic at $q=0$.

An automorphic form $f(z)$ with respect to $G$ is called a cusp form with respect to $G$, if the following conditions are satisied.

$\left(\mathrm{A} 1^{\prime}\right) f(z)$ is holomorphic on $\mathcal{H}$.

(A $\left.3^{\prime}\right)$ For every cusp s of $G$, the function $g(q)$, defined as above, is holomorphic and takes the value 0 at $q=0$. 
We denote by $S_{\nu}(G)$ the set of all cusp forms of degree $\nu$ with respect to $G$. In this paper we shall only deal with the forms of even degree.

\section{$\S 2$. $M_{n}$-forms and $M_{n}$-vectors.}

Let

$$
G L(2, \boldsymbol{C}) \ni \sigma \rightarrow M_{n}(\sigma) \in G L(n+1, \boldsymbol{C})
$$

be the representation of $G L(2, \boldsymbol{C})$ by symmetric contravariant tensors of order $n$, so that the equality

$$
\sigma\left(\begin{array}{l}
u \\
v
\end{array}\right)=\left(\begin{array}{c}
z \\
w
\end{array}\right)
$$

is led to

$$
M_{n}(\sigma)\left(\begin{array}{c}
u^{n} \\
u^{n-1} v \\
\vdots \\
u v^{n-1} \\
v^{n}
\end{array}\right)=\left(\begin{array}{c}
z^{n} \\
z^{n-1} w \\
\vdots \\
z w^{n-1} \\
w^{n}
\end{array}\right)
$$

For instance, we have

$$
M_{n}\left(\left(\begin{array}{cc}
1 & z \\
0 & 1
\end{array}\right)\right)=\left(\begin{array}{rrrrr}
1 & n z \frac{n(n-1)}{2} z^{2} & \cdots & z^{n} \\
0 & 1 & (n-1) z & \cdots & z^{n-1} \\
& \cdots & & \cdots & \\
0 & \cdots & & \cdots & 1
\end{array}\right) .
$$

As this matrix $M_{n}\left(\left(\begin{array}{ll}1 & z \\ 0 & 1\end{array}\right)\right)$ will be often used in our investigation, we denote it briefly by $L_{n}(z)$ :

$$
L_{n}(z)=M_{n}\left(\left(\begin{array}{ll}
1 & z \\
0 & 1
\end{array}\right)\right) \text {. }
$$

We have then, for every $\tau \in S L(2, \boldsymbol{R})$,

$$
L_{n}(\tau(z))^{-1} M_{n}(\tau) L_{n}(z)=M_{n}\left(\left(\begin{array}{cc}
J & 0 \\
c & J^{-1}
\end{array}\right)\right),
$$

where $J=J(\tau, z)=(c z+d)^{-1}$. In particular, if $r$ is a real number and $\tau=\left(\begin{array}{ll}1 & r \\ 0 & 1\end{array}\right)$, we have

$$
L_{n}(\tau(z))=M_{n}(\tau) L_{n}(z)
$$

Let $f$ be an automorphic form of degree $n+2$ with respect to $G$. In [3] we have studied the vector differential form

$$
\omega=L_{n}(z)\left(\begin{array}{c}
0 \\
\vdots \\
0 \\
f
\end{array}\right) d z=\left(\begin{array}{c}
f z^{n} d z \\
f z^{n-1} d z \\
\vdots \\
f d z
\end{array}\right)
$$


which satisfies, for every $\sigma \in G$,

$$
\omega \circ \sigma=M_{n}(\sigma) \omega .
$$

This is an example of $M_{n}$-form, whose definition is given as follows. A column vector of dimension $n+1$

$$
\omega=\left(\begin{array}{c}
\omega_{0} \\
\omega_{1} \\
\vdots \\
\omega_{n}
\end{array}\right)
$$

is called an $M_{n}$-form with respect to $G$, if it satisfies the following conditions (M1-3).

(M1) Each component $\omega_{k}$ is a meromorphic differential form on $\mathcal{H}$.

(M2) For every $\sigma \in G$, we have $\omega \circ \sigma=M_{n}(\sigma) \omega$.

Let $s$ be a cusp of $G$; and let $\rho$ and $h$ be as in $\S 1$. Then, if $\omega$ satisfies (M $1-2)$, we can easily verify, using (4), that the form

$$
L_{n}(z)^{-1} M_{n}(\rho)^{-1} \omega \circ \rho
$$

is invariant under the translation $z \rightarrow z+h$. Therefore, if we put $q=\exp \left(2 \pi i h^{-1} z\right)$, there exist $n+1$ functions $f_{0}(q), \cdots, f_{n}(q)$, meromorphic in $0<|q|<1$, such that

$$
L_{n}(z)^{-1} M_{n}(\rho)^{-1} \omega \circ \rho=\left(\begin{array}{c}
f_{0}(q) d q \\
\vdots \\
f_{n}(q) d q
\end{array}\right) .
$$

Now the condition (M 3 ) is stated as follows.

(M3) For every cusp $s$ of $G$, the functions $f_{k}(q)$ defined by (6) are meromorphic at $q=0$.

An $M_{n}$-form $\omega$ with respect to $G$ is called a cusp $M_{n}$-form with respect to $G$ if the following conditions ( $\left(\mathrm{M}^{\prime}\right)$ and $\left(\mathrm{M} 3^{\prime}\right)$ are satisfied.

$\left(\mathrm{M} 1^{\prime}\right)$ Every component of $\omega$ is holomorphic on $\mathcal{H}$.

$\left(\mathrm{M}^{\prime}\right)$ For every cusp $s$ of $G$, the functions $f_{k}(q)$ defined by (6) are holomorphic at $q=0$.

We can prove that the form $\omega$ defined by (5) is an $M_{n}$-form with respect to $G$; it is a cusp $M_{n}$-form if and only if $f(z)$ is a cusp form. This fact is a special case of the following Theorem 1. We shall denote by $\mathfrak{F}_{n}(G)$ the set of all cusp $M_{n}$-forms with respect to $G$.

Considering functions in place of differential forms, we get the following definition. A column vector of dimension $n+1$

$$
\mathfrak{g}=\left(\begin{array}{c}
g_{0} \\
\vdots \\
g_{n}
\end{array}\right)
$$

is called an $M_{n}$-vector with respect to $G$, if it satisfies the following conditions 
(V 1-3).

(V1) Every component $g_{k}$ is a meromorphic function on $\mathcal{H}$.

(V 2) For every $\sigma \in G$, we have go $\sigma=M_{n}(\sigma) \mathrm{g}$.

The notations $s, \rho, h$ being as above, if $g$ satisfies (V 1-2), there exist $n+1$ functions $F_{0}(q), \cdots, F_{n}(q)$, meromorphic in $0<|q|<1$, such that

$$
L_{n}(z)^{-1} M_{n}(\rho)^{-1} \mathfrak{g} \circ \rho=\left(\begin{array}{c}
F_{0}(q) \\
\vdots \\
F_{n}(q)
\end{array}\right) .
$$

(V3) For every cusp s of $G$, the functions $F_{k}(q)$ defined by (7) are meromorphic at $q=0$.

An $M_{n}$-vector $g$ with respect to $G$ is called a cusp $M_{n}$-vector with respect to $G$ if the following conditions ( $\left.\mathrm{V} 1^{\prime}\right)$ and $\left(\mathrm{V} 3^{\prime}\right)$ are satisfied.

$\left(\mathrm{V} 1^{\prime}\right)$ Every component of $\mathfrak{g}$ is holomorphic on $\mathcal{H}$.

$\left(\mathrm{V} 3^{\prime}\right)$ For every cusp s of $G$, the functions $F_{k}(q)$ defined by (7) are holomorphic and take the value 0 at $q=0$.

We shall denote by $\mathfrak{B}_{n}(G)$ the set of all cusp $M_{n}$-vectors with respect to $G$.

\section{§3. Main results.}

We shall now state our results in the following theorems, for which the proofs will be given in $\S 4$. We first introduce some notations. For every integer $k$ and a non-negative integer $j$, we shall write

$$
\left(\begin{array}{c}
k \\
j
\end{array}\right)=\left\{\begin{array}{cl}
1 & \text { for } j=0, \\
\frac{k(k-1) \cdots(k-j+1)}{j !} & \text { for } j>0 .
\end{array}\right.
$$

Consider a triplet $(n, \nu, k)$ of integers such that

i) $n$ is even and non-negative;

ii) $\nu$ is even and $-(n-2) \leqq \nu \leqq n+2$;

iii) $0 \leqq k \leqq n-\frac{\nu+n-2}{2}$.

For such a triplet $(n, \nu, k)$, we put

$$
\alpha_{n, \nu, k}=\left\{\begin{array}{cl}
0 & \text { for } \nu+k-1<0 . \\
\left(k+\frac{\nu+n-2}{2}\right) ! & \text { for } \nu+k-1 \geqq 0
\end{array}\right.
$$

and

$$
r_{n, \nu, k}=\left\{\begin{array}{cl}
0 & \text { for } \nu+k-1<0, \\
\frac{\left(k+\frac{\nu+n}{2}\right) !}{k !(\nu+k-1) !} & \text { for } \nu+k-1 \geqq 0 .
\end{array}\right.
$$


For fixed $n$ and $\nu$, we denote $\alpha_{n, \nu, k}$ and $\gamma_{n, \nu, k}$ simply by $\alpha_{k}$ and $\gamma_{k}$.

LeMma 1. Let $t$ be an integer such that $0 \leqq t \leqq n$ and $f_{t}, f_{t+1}, \cdots, f_{n}$ be $n-t+1$ meromorphic functions on $\mathcal{H}$. If

$$
\omega=L_{n}(z)\left(\begin{array}{c}
0 \\
\vdots \\
0 \\
f_{t} \\
\vdots \\
f_{n}
\end{array}\right) d z
$$

is an $M_{n}$-form with respect to $G$, then $f_{t}$ is an automorphic form of degree $2 t+2-n$ with respect to $G$. Moreover, if $\omega$ is a cusp $M_{n}$-form, $f_{t}$ is a cusp form.

THEOREM 1. Let $n$ and $\nu$ be two even integers such that $n>0$ and $-(n-2) \leqq$ $\nu \leqq n+2 ;$ put $\mu=\frac{n+2-\nu}{2}$. Then, for every automorphic form $f$ of degree $\nu$ with respect to $G$, the vector differential form

$$
\omega=L_{n}(z)\left(\begin{array}{c}
0 \\
\vdots \\
0 \\
\alpha_{0} f \\
\alpha_{1} f^{\prime} \\
\vdots \\
\alpha_{\mu} f^{(\prime)}
\end{array}\right) d z
$$

is an $M_{n}$-form with respect to $G$, where $\alpha_{0}=\alpha_{n, \nu, 0}, \cdots, \alpha_{k}=\alpha_{n, \nu, k} ; f^{\prime}, \cdots, f^{(\mu)} d e$ note the derivatives $d f / d z, \cdots, d^{\prime \prime} f / d z^{\prime \prime}$; and the number of 0 in the column is $n-\mu$. Moreover, in order that $\omega$ is a cusp $M_{n}$-form, it is necessary and sufficient that $f$ is a cusp form.

Remark that, if $\nu \leqq 0$, we have $\alpha_{0}=\alpha_{1}=\cdots=\alpha_{-\nu}=0$. We denote by $\mathfrak{S}^{n}{ }_{\nu}(G)$ the set of all $M_{n}$-forms $\omega$ of the form (8), where $f$ is a cusp form of degree $\nu$. If $\nu \leqq 0$, the set $\mathbb{S}^{n}{ }_{\nu}(G)$ consists only of the zero element. If $\nu>0$, we have $\alpha_{0} \neq 0$, so that the vector space $\widetilde{S}^{n}{ }_{\nu}(G)$ is canonically isomorphic to the vector space $S_{\nu}(G)$ by the mapping $f \rightarrow \omega$.

THEOREM 2. The vector space $\mathfrak{\mho}_{n}(G)$ of all cusp $M_{n}$-forms is the direct sum of the vector spaces $\mathfrak{S}_{\nu}^{n}(G)$ for even $\nu$ such that $2 \leqq \nu \leqq n+2$ :

$$
\mathfrak{\mho}_{n}(G)=\bigodot^{n}{ }_{2}(G)+\cdots+\subseteq^{n}{ }_{n}(G)+\mathfrak{S}^{n}{ }_{n+2}(G) .
$$

Hence, if we denote by $d_{\nu}(G)$ the dimension of the vector space $S_{\nu}(G)$, the dimension of the vector space $\mathfrak{F}_{n}(G)$ is equal to

$$
d_{2}(G)+\cdots+d_{n}(G)+d_{n+2}(G) .
$$

The number $d_{\nu}(G)$ is easily obtained by means of Riemann-Roch Theorem. 
We note that from Lemma 1 and Theorem 1 follows a result of Bol [1], which asserts the $(n-1)$-th derivative of an automorphic form of degree $-(n-2)$ to be an automorphic form of degree $n$. In fact, consider the case $\nu=-(n-2)$ in Theorem 1; we have then

$$
\alpha_{0}=\alpha_{1}=\cdots \alpha_{n-2}=0, \alpha_{n-1} \neq 0 ;
$$

so the vector

$$
L_{n}(z)\left(\begin{array}{c}
0 \\
\vdots \\
0 \\
\alpha_{n-1} f^{(n-1)} \\
\alpha_{n} f^{(n)}
\end{array}\right) d z
$$

is an $M_{n}$-form for every automorphic form $f$ of degree $-(n-2)$. Hence, by Lemma 1, $f^{(n-1)}$ is an automorphic form of degree $n$.

TheOREM 3. Let the integers $n, \nu, \mu$ be the same as in Theorem 1 . Then, for every automorphic form $f$ of degree $\nu$ with respect to $G$, the vector function

$$
\mathfrak{f}=L_{n}(z)\left(\begin{array}{c}
0 \\
\vdots \\
0 \\
\gamma_{0} f \\
\gamma_{1} f^{\prime} \\
\vdots \\
\gamma_{\mu-1} f^{(\mu-1)}
\end{array}\right)
$$

is an $M_{n}$-vector with respect to $G$, where $\gamma_{k}=\gamma_{n, v, k}$; and the number of 0 in the column is $n-\mu+1$. Moreover, in order that $\mathfrak{f}$ is a cusp $M_{n}$-vector, it is necessary and sufficient that $f$ is a cusp form.

Denote by $\mathfrak{T}_{\nu}^{n}(G)$ the set of all $M_{n}$-vectors $\mathfrak{f}$ of the form (9), where $f$ is a cusp form of degree $\nu$. We see easily $\mathfrak{T}_{\nu}^{n}(G)=\{0\}$ for $\nu \leqq 0$ and $\nu=n+2$. If $0<\nu \leqq n$, we have $\gamma_{0} \neq 0$, so that the vector space $\mathfrak{I}_{\nu}^{n}(G)$ is canonically isomorphic to the vector space $S_{\nu}(G)$ by the mapping $f \rightarrow \mathfrak{f}$.

THEOREM 4. The vector space $\mathfrak{B}^{n}(G)$ of all cusp $M_{n}$-vectors is the direct sum of the vector spaces $\mathfrak{I}_{\nu}^{n}(G)$ for even $\nu$ such that $2 \leqq \nu \leqq n$ :

$$
\mathfrak{B}^{n}(G)=\mathfrak{T}_{2}^{n}(G)+\cdots+\mathfrak{I}_{n}^{n}(G) .
$$

Now we consider the differential $d \mathfrak{j}$ of an $M_{n}$-vector $\mathfrak{f}$. If $\mathfrak{f}$ is an $M_{n}$ vector with respect to $G$, then we can easily prove that $d \mathfrak{f}$ is an $M_{n}$-form with respect to $G$; if $\mathfrak{f}$ is a cusp $M_{n}$-vector, then $d \mathfrak{f}$ is a cusp $M_{n}$-form. More precisely, we have

THEOREM 5. The integers $n, \nu, \mu$ being as in Theorem 1 , let $f$ be an auto- 
morphic form of degree $\nu$ with respect to $G$. Define an $M_{n}$-form $\omega$ and an $M_{n^{-}}$ vector $\mathrm{f}$ by (8) and (9). Then we have

$$
d \mathfrak{\uparrow}=\mu(n-\mu+1) \omega .
$$

Remark that $\mu(n-\mu+1) \neq 0$ if $\mu \geqq 1$. Hence, if $0<\nu \leqq n$, the mapping $\mathfrak{\uparrow} \rightarrow d \mathfrak{f}$ gives an isomorphism of $\mathfrak{I}_{\nu}^{n}(G)$ onto $\mathfrak{S}_{\nu}^{n}(G)$.

From the last theorem, we can conclude that, if $0<\nu<n+2$ and if $\omega \in$ $\Im^{n}{ }_{\nu}(G)$, the period of the integral $\int^{z} \omega$ is cohomologous to 0 in the sense of [3]. On the other hand, Theorem 1 of [3] claims that the period of $\int^{z} \omega$ is not cohomologous to 0 for every element $\omega \neq 0$ of $\widetilde{S}_{n+2}^{n}(G)$. Therefore, we obtain the following result.

THEOREM 6 . Let $\mathfrak{R}_{n}(G)$ denote the set of all cusp $M_{n}$-forms with respect to $G$, whose integrals have the periods cohomologous to 0 . Then, the factor space $\mathfrak{F}_{n}(G) / \mathfrak{N}_{n}(G)$ is canonically isomorphic to $S_{n+2}(G)$.

Put, similarly as in [3], for $\omega, \eta \in \mathfrak{F}_{n}(G)$,

$$
(\omega, \eta)=i \int_{\mathscr{D}}{ }^{t} \omega P_{n} \bar{\eta},
$$

where $P_{n}$ is the symmetric matrix introduced in $\S 1$ of [3] and $\mathscr{D}$ is a fundamental domain of $G$. Then $(\omega, \eta)$ is a Hermitian form on $\mathfrak{F}_{n}(G)$. By the above considerations, we see that two subspaces $\mathbb{S}_{n+2}^{n}(G)$ and $\mathbb{S}_{2}^{n}(G)+\cdots+\mathbb{S}_{n}^{n}(G)$ of $\mathfrak{F}_{n}(G)$ are transversal to each other with respect to this form $(\omega, \eta)$, and $(\omega, \eta)$ is a zero form on the latter space, while it is a definite form on the former space ( $\$ 2$ of [3]).

\section{$\S 4$. Proofs of Theorems.}

Lemma 2. Let $f_{0}, \cdots, f_{n}$ be $n+1$ meromorphic functions on $\mathcal{H}$; put

$$
\mathfrak{i}=\left(\begin{array}{c}
f_{0} \\
\vdots \\
f_{n}
\end{array}\right), \omega=L_{n}(z) \mathfrak{f} d z .
$$

Then, $\omega$ satisfies the condition (M 2) if and only if

$$
(\mathfrak{f} \circ \sigma) J^{2}=M_{n}\left(\left(\begin{array}{cc}
J & 0 \\
c & J^{-1}
\end{array}\right)\right) \mathfrak{f}
$$

holds for every $\sigma=\left(\begin{array}{ll}a & b \\ c & d\end{array}\right) \in G$, where $J=J(\sigma, z)=(c z+d)^{-1}$.

This follows from the relation (3) of $\S 2$.

Let $\tau=\left(\begin{array}{ll}a & b \\ c & d\end{array}\right)$ be an element of $S L(2, \boldsymbol{R})$ and $J=(c z+d)^{-1}$; we have then 


$$
M_{n}\left(\left(\begin{array}{cc}
J & 0 \\
c & J^{-1}
\end{array}\right)\right)=J^{n}\left(\begin{array}{cccc}
1 & & & \\
c J^{-1} & J^{-2} & & \\
\cdots & \cdots & \cdots & \\
c^{n} J^{-n} & n c^{n-1} J^{-n-1} & \cdots & J^{-2 n}
\end{array}\right) .
$$

In the matrix (10), the elements above the diagonal are all 0 ; the $(r+1)$-th diagonal element is $J^{n-2 r}$; and the $(r+1)$-th row is

$$
\left(c^{r} J^{n-r},\left(\begin{array}{c}
r \\
1
\end{array}\right) c^{r-1} J^{n-r-1},\left(\begin{array}{c}
r \\
2
\end{array}\right) c^{r-2} J^{n-r-2}, \cdots, J^{n-2 r}, 0, \cdots, 0\right) .
$$

We shall now prove Lemma 1. Suppose that $f_{0}=\cdots=f_{t-1}=0$ in Lemma 2 and $\omega=L_{n}(z)$ f $d z$ is an $M_{n}$-form with respect to $G$. Then, by Lemma 2 and by (10), we have, for every $\sigma \in G$,

$$
\left(f_{t}^{\circ} \sigma\right) J(\sigma, z)^{2 t+2-n}=f_{t}
$$

so $f_{t}$ satisfies the condition (A 2) for $\nu=2 t+2-n$. Let $s$ be a cusp of $G ; \rho, h$ and $q$ being defined for $s$ as in $\S 2$, there exist $n+1$ meromorphic functions $g_{0}(q), \cdots, g_{n}(q)$ in $|q|<1$, such that

$$
L_{n}(z)^{-1} M_{n}(\rho)^{-1} L_{n}(\rho(z))(\mathfrak{f} \circ \rho) J(\rho, z)^{2} d z=\left(\begin{array}{c}
g_{0}(q) d q \\
\vdots \\
g_{n}(q) d q
\end{array}\right) .
$$

By the relation (3), putting $J=J(\rho, z)=(c z+d)^{-1}$, we have

so that by (10),

$$
M_{n}\left(\left(\begin{array}{cc}
J & 0 \\
c & J^{-1}
\end{array}\right)\right)^{-1}\left(\mathfrak{f}_{\circ} \rho\right) J^{2}=2 \pi i h^{-1} q\left(\begin{array}{c}
g_{0}(q) \\
\vdots \\
g_{n}(q)
\end{array}\right)
$$

$$
\left(f_{t}^{\circ} \rho\right) J^{2 t+2-n}=2 \pi i h^{-1} q g_{t}(q) .
$$

This shows that $f_{t}$ satisfies (A 3). Hence $f_{t}$ is an automorphic form of degree $2 t+2-n$ with respect to $G$. Furthermore, if $\omega$ is a cusp $M_{n}$-form, $f_{t}$ must be holomorphic on $\mathscr{G}$, since $f_{t}$ is the $(t+1)$-th component of $L_{n}(-z) \omega / d z$; and as $g_{t}(q)$ is holomorphic at $q=0$ by virtue of (M $\left.3^{\prime}\right)$, the relation (11) shows that $f_{t}$ satisfies $\left(\mathrm{A} 3^{\prime}\right)$. This completes the proof of Lemma 1.

Lemma 3. If $f$ is an automorphic form of degree $\nu$ with respect to $G$, we have, for every $\sigma=\left(\begin{array}{ll}a & b \\ c & d\end{array}\right) \in G$,

$$
\left(f^{(k)} \circ \sigma\right) J^{2}=\sum_{j=0}^{k}\left(\begin{array}{c}
k \\
j
\end{array}\right)\left(\begin{array}{c}
\nu+k-1 \\
j
\end{array}\right) j ! c^{j j J^{j+2-2 k-\nu} f^{(k-j)}},
$$

where $J=J(\sigma, z)=(c z+d)^{-1}$.

This is easily obtained by the induction on $k$.

Now we shall prove Theorem 1. Notations being as in that theorem, by 
Lemma 2, $\omega$ satisfies the condition (M2) if we have, for every $\sigma \in G$,

$$
J^{2}\left(\begin{array}{c}
0 \\
\vdots \\
0 \\
\alpha_{0} f_{\circ \sigma} \\
\alpha_{1} f^{\prime \circ \sigma} \\
\vdots \\
\alpha_{\mu} f^{(\mu)} \circ \sigma
\end{array}\right)=M_{n}\left(\left(\begin{array}{cc}
J & 0 \\
c & J^{-1}
\end{array}\right)\right)\left(\begin{array}{c}
0 \\
\vdots \\
0 \\
\alpha_{0} f \\
\alpha_{1} f^{\prime} \\
\vdots \\
\alpha_{\mu} f^{(\mu)}
\end{array}\right),
$$

where $J=J(\sigma, z)=(c z+d)^{-1}$. Put $t=n-\mu$. By (10), we see that the first $t$ components of the vectors in both sides are equal to 0 ; and by $\left(10^{\prime}\right)$, if $r \geqq t$, the $(r+1)$-th component of the vector on the right hand side of (12) is equal to

$$
\sum_{u=t}^{r}\left(\begin{array}{l}
r \\
u
\end{array}\right) c^{r-u} j^{n-r-u} \alpha_{u-t} f^{(u-t)} ;
$$

hence the equality (12) is proved if we show that (13)] is equal to $J^{2} \alpha_{r-t} f^{(r-t)} \circ \sigma_{\circ}$ By Lemma 3, we have

$$
\begin{aligned}
J^{2} \alpha_{r-t} f^{(r-t)} \circ \sigma & =\alpha_{r-t} \sum_{j=0}^{r-t}\left(\begin{array}{c}
r-t \\
j
\end{array}\right)\left(\begin{array}{c}
\nu+r-t-1 \\
j
\end{array}\right) j ! c^{j j} J^{j+2-2(r-t)-\nu} f^{(r-t-j)} \\
& =\alpha_{r-t} \sum_{u=t}^{r}\left(\begin{array}{c}
r-t \\
r-u
\end{array}\right)\left(\begin{array}{c}
\nu+r-t-1 \\
r-u
\end{array}\right)(r-u) ! c^{r-u j e(u)} f^{(u-t)},
\end{aligned}
$$

where $e(u)=r-u+2-2(r-t)-\nu$. Since $\nu=2 t-(n-2)$, we have $e(u)=n-r-u$. On the other hand, we can easily verify

$$
\alpha_{r-t}\left(\begin{array}{c}
r-t \\
r-u
\end{array}\right)\left(\begin{array}{c}
\nu+r-t-1 \\
r-u
\end{array}\right)(r-u) !=\alpha_{u-t}\left(\begin{array}{l}
r \\
u
\end{array}\right) .
$$

This proves the equality (10). Hence $\omega$ satisfies (M2). The condition (M.1) is of course satisfied. Now consider a cusp $s$ of $G$. Since $\omega$ satisfies (M 1-2), $\rho$ and $q$ being as in $\S 1$, there exist $n+1$ meromorphic functions $f_{0}(q), \cdots, f_{n}(q)$ in $0<|q|<1$ such that

$$
L_{n}(z)^{-1} M_{n}(\rho)^{-1} \omega \circ \rho=\left(\begin{array}{c}
f_{0}(q) d q \\
\vdots \\
f_{n}(q) d q
\end{array}\right) .
$$

By (A 3), there exists a meromorphic function $g(q)$ in $|q|<1$ such that

$$
f(\rho(z))=g(q) J(\rho, z)^{-\nu} .
$$

Differentiating this successively, we get, for every $k$,

$$
f^{(k)}(\rho(z))=J^{a(k)} \sum_{u} F_{k u}(q) z^{u},
$$


where $a(k)$ is an integer and the $F_{k u}(q)$ are meromorphic functions in $|q|<1$. Comparing both sides of the equality

$$
\left(\begin{array}{c}
f_{0}(q) d q \\
\vdots \\
f_{n}(q) d q
\end{array}\right)=L_{n}(z)^{-1} M_{n}(\rho)^{-1} L_{n}(\rho(z))\left(\begin{array}{c}
0 \\
\vdots \\
0 \\
\alpha_{0} f \circ \rho \\
\vdots \\
\alpha_{\mu} f^{(\mu)} \circ \rho
\end{array}\right) J^{2} \frac{h}{2 \pi i} \frac{d q}{q},
$$

we observe that $f_{k}(q)$ is written in the form

$$
f_{k}(q)=J^{b(k)} \sum_{u} H_{k u}(q) z^{u}
$$

where $b(k)$ is an integer and the $H_{k u}(q)$ are meromorphic functions in $|q|<1$. Hence there exists an integer $m$ such that

$$
\lim _{q \rightarrow 0} q^{m} f_{k}(q)=0
$$

for every $k$. This shows that the $f_{k}(q)$ are meromorphic at $q=0$. Thus we have proved that $\omega$ is an $M_{n}$-form. Furthermore, suppose that $f$ is a cusp form. Then the function $g(q)$ of (14) takes the value 0 at $q=0$; so, in the expression (15), we may assume that the $F_{k u}(q)$ take the value 0 at $q=0$. Comparing again both sides of (16), we see that the functions $H_{k u}(q)$ in the expression [17) are holomorphic at $q=0$, so that we have

$$
\lim _{q \rightarrow 0} q f_{k}(q)=0
$$

for every $k$. This shows that the $f_{k}(q)$ are holomorphic at $q=0$. Hence $\omega$ is a cusp $M_{n}$-form. We can similarly show that if $\omega$ is a cusp $M_{n}$-form, $f$ satisfies $\left(\mathrm{A}^{\prime}\right)$. Theorem 1 is then completely proved.

We can prove Theorem 3 in a quite similar way. We shall now prove Theorem 5, Differentiating both sides of

$$
L_{n}(z+w)=L_{n}(z) L_{n}(w)
$$

with respect to $w$, and then putting $w=0$, we obtain

$$
L_{n}{ }^{\prime}(z)=L_{n}(z) L_{n}{ }^{\prime}(0) \text {. }
$$

From (2) we see that

$$
L_{n}{ }^{\prime}(0)=\left(\begin{array}{cccccc}
0 & n & 0 & \cdots & 0 & 0 \\
0 & 0 & n-1 & \cdots & 0 & 0 \\
& \cdots & & \cdots & \cdots & \\
0 & \cdots & & \cdots & 2 & 0 \\
0 & \cdots & & \cdots & 0 & 1 \\
0 & \cdots & & \cdots & 0 & 0
\end{array}\right)
$$


Notations being as in Theorem 5, we have, using (18) and (19),

$$
\begin{aligned}
& d \tilde{f}=d\left[L_{n}(z)\left(\begin{array}{c}
0 \\
\vdots \\
0 \\
\gamma_{0} f \\
\vdots \\
\gamma_{\mu-1} f^{(\mu-1)}
\end{array}\right)\right]=\left[L_{n}^{\prime}(z)\left(\begin{array}{c}
0 \\
\vdots \\
0 \\
\gamma_{0} f \\
\vdots \\
\gamma_{\mu-1} f^{(\mu-1)}
\end{array}\right)+L_{n}(z)\left(\begin{array}{c}
0 \\
\vdots \\
0 \\
\gamma_{0} f^{\prime} \\
\vdots \\
\gamma_{\mu-1} f^{(\mu)}
\end{array}\right)\right] d z \\
& =L_{n}(z)\left(\begin{array}{c}
\alpha_{0}^{\prime} f \\
\alpha_{1}^{\prime} f^{\prime} \\
\vdots \\
\alpha_{\mu}^{\prime} f^{(\mu)}
\end{array}\right) d z
\end{aligned}
$$

where $\alpha_{0}{ }^{\prime}=\mu \gamma_{0}, \alpha_{1}{ }^{\prime}=(\mu-1) \gamma_{1}+\gamma_{0}, \cdots, \alpha_{\mu-1}{ }^{\prime}=\gamma_{\mu-1}+\gamma_{\mu-2}, \alpha_{\mu}{ }^{\prime}=\gamma_{\mu-1}$. We can easily verify $\alpha_{k}{ }^{\prime}=\mu(n-\mu+1) \alpha_{k}$ for $0 \leqq k \leqq \mu$. This proves Theorem 5 .

It remains to prove Theorem 2 and Theorem 4. We need for that purpose

Lemma 4. Suppose that the Fuchsian group $G$ has no cusp. Let $n$ be a positive even integer and $r=\frac{n}{2}$. Then there is no cusp $M_{n}$-form $\omega$ with respect to $G$ of the type

$$
\omega=L_{n}(z)\left(\begin{array}{c}
0 \\
\vdots \\
0 \\
1 \\
f_{r} \\
\vdots \\
f_{n}
\end{array}\right) d z,
$$

where $f_{r}, \cdots, f_{n}$ are meromorphic functions on $\mathcal{H}$.

Proof. First we remark that $f_{r}$ must be everywhere holomorphic on $\mathscr{A}$. By lemma 2 and by $\left(10^{\prime}\right)$, we have, for every $\sigma=\left(\begin{array}{ll}a & b \\ c & d\end{array}\right) \in G$,

$$
f_{r}(\sigma(z)) J(\sigma, z)^{2}=f_{r}(z)+r c J(\sigma, z) .
$$

Put $\eta=f_{r}(z) d z$. Then $\eta$ is a holomorphic differential form on $\mathscr{G}$ satisfying

$$
\eta \circ \sigma=\eta-r d(\log J(\sigma, z))
$$

for every $\sigma \in G$. Consider the integral of $\eta$ along the boundary $\mathcal{B}$ of a fundamental domain of $G$; then we find, taking account of the relation (20), 


$$
\frac{2}{r} \int_{\mathcal{B}} \eta=2 g-2+\sum_{\lambda}\left(1-\frac{1}{m_{\lambda}}\right)
$$

where $g$ is the genus of the Riemann surface $\mathscr{H} / G$ and the $m_{\lambda}$ denote the orders of ramification at the elliptic points of $G$. It is well known that the number on the right hand side of (21) is positive. On the other hand, as $\eta$ is holomorphic, we must have $\int_{\mathscr{B}} \eta=0$; thus we are led to contradiction if we assume the existence of a cusp $M_{n}$-form of the type described in our lemma.

Now we are ready to prove Theorem 2. First we remark that $S_{\nu}(G)=\{0\}$ for $\nu<0$ and $S_{0}(G)=\{0\}$ or $=C$ according as $G$ has a cusp or not. Let $\omega$ be a cusp $M_{n}$-form with respect to $G$; put

$$
L_{n}(z)^{-1} \omega=\left(\begin{array}{c}
f_{0}(z) \\
\vdots \\
f_{n}(z)
\end{array}\right) d z .
$$

Let $t$ be the first integer such that $f_{t} \neq 0$. Then, by Lemma $1, f_{t}$ is a cusp form with respect to $G$ of degree $2 t-n+2$. By the above remark, we must have $t \geqq \frac{n-2}{2}$. If $t=\frac{n-2}{2}, f_{t}$ is a cusp form of degree 0 ; then, $G$ has no cusp and $f_{t}$ is a constant. This is impossible, however, in view of Lemma 4 . Hence we have $2 t-n+2>0$. Put $\nu=2 t-n+2$. Then we have $\alpha_{n, \nu, 0} \neq 0$; put $f=\alpha_{n, \nu, 0^{-1}} f_{t}$. Let $\eta_{\nu}$ be the cusp $M_{n}$-form defined for the cusp form $f$ by (8). Then we see that the first $t+1$ components of $L_{n}(z)^{-1}\left(\omega-\eta_{\nu}\right)$ are all 0 . Applying the same argument to the form $\omega-\eta_{\nu}$, we can find an element $\eta_{\nu+2}$ of $\widetilde{S}_{\nu+2}^{n}(G)$ such that the first $t+2$ components of $L_{n}(z)^{-1}\left(\omega-\eta_{\nu}-\eta_{\nu+2}\right)$ are all 0. Repeating this procedure, we get the expression

$$
\omega=\sum_{\lambda=\nu}^{n+2} \eta_{\lambda}
$$

where $\eta_{\lambda}$ is an element of $\widetilde{S}_{\lambda}^{n}(G)$ for every $\lambda$. It is easy to see that this expression gives a decomposition of the vector space $\widetilde{\mho}_{n}(G)$ as the direct sum of the vector spaces $\mathfrak{S}_{\lambda}^{n}(G)$ for $2 \leqq \lambda \leqq n+2$. Thus we have proved Theorem 2. Theorem 4 can be $\mathcal{S}^{n}{ }_{\lambda}$ proved in a quite similar way.

University of Tokyo.

\section{References}

[1] Bol, G., Invarianten linearer Differentialgleichungen, Abh, Math. Seminar Hamburger Univ., 16 (1948), 1-28.

[2] Eichler, M., Eine Verallgemeinerung der Abelschen Integrale, Math. Z., 67 (1957), 267-298.

[3] Shimura, G., Sur les intégrales attachées aux formes automorphes, J. Math. Soc. Japan, 11 (1959), 291-311. 